Artículo de investigación.

Cómo citar: E. Velásquez, y G. Forero. “Simulación hidrológica para sistemas de drenaje sostenible aplicada en jardines verticales en el humedal La Vaca". Inventum, vol. 15 , no. 28 , pp. 88-103. doi: 10.26620/uniminuto.inventum.15.28.2020.88-103

Editorial: Corporación Universitaria

Minuto de Dios - UNIMINUTO.

ISSN: $1909-2520$

eISSN: $2590-8219$

Fecha de recibido: 10 de enero del 2020 Fecha de aprobado: 20 de marzo del 2020 Fecha de publicación: 30 de mayo del 2020

Conflicto de intereses: los autores han declarado que no existen intereses en competencia.

Edward Daniel Velásquez García evelasquez@unbosque.edu.co ORCID: 0000-0001-9469-7475 Universidad El Bosque, Colombia

Gonzalo Alberto Forero Buitrago gonzalo.forero@mail.escuelaing.edu.co ORCID: 0000-0002-9739-4741

Universidad El Bosque, Colombia

Gina Paola González Angarita ginap.gonzaleza@unilibrebog.edu.co ORCID: 0000-0002-9436-5371

Universidad Libre, Colombia

\section{Simulación hidrológica para sistemas de drenaje sostenible aplicada en jardines verticales en el humedal La Vaca, Bogotá D.C.}

\author{
Hydrological simulation for sustainable \\ drainage systems applied to vertical \\ gardens in La Vaca wetland, Bogotá D.C.
}

\author{
Simulação hidrológica para drenagem \\ sustentável aplicada em jardins vertical \\ no pantanal La Vaca, Bogotá D.C.
}

\begin{abstract}
Resumen
Los Sistemas Urbanos de Drenaje Sostenible (SUDS) en la zona de influencia del humedal La Vaca son una alternativa para promover el uso eficiente del recurso hídrico. Para su simulación se proponen tres componentes principales: evaluación de las potenciales áreas de captación de agua lluvia, optimización de las condiciones de operación hidráulica para un jardín vertical en Corabastos de acuerdo a la hidrología y por último la simulación del sistema hidráulico propuesto en el software Pipe Flow Expert. Los resultados de la simulación sugieren que implementar los SUDS sobre las cubiertas de Corabastos son técnicas factibles dado que no existe ningún periodo de completa sequia durante el año en la localidad de Kennedy; esto permitirá obtener cerca de 1'537.000 litros de agua lluvia anual que podría ser aprovechada de marzo a noviembre sin déficit, cubriría las necesidades de jardines verticales en la zona y añadiría disponibilidad para otros usos. Finalmente, se concluye que este tipo de sistemas permiten mitigar las afectaciones a la infraestructura inundable con enormes beneficios sociales, paisajísticos y ecológicos.
\end{abstract}

Palabras clave: Aprovechamiento pluvial, Flow Master, humedal, humedal La Vaca, inundaciones, Pipe Flow Expert, riesgo, Sistema urbano de drenaje sostenible.

\begin{abstract}
Sustainable Drainage Systems (SuDS) in the area of influence of the La Vaca wetland are an alternative to promote the efficient use of water resources. For its modeling, three main components are proposed: Evaluation of rainwater harvesting areas, Hydraulic optimization for a vertical garden in Corabastos according to the hydrology and finally the Simulation of the proposed hydraulic system in the software Pipe Flow Expert. The modeling results suggest that implementing the SUDS on the Corabastos covers are feasible techniques since there is no period of complete drought during the year in the Kennedy locality; Obtaining close to 1,537,000 liters of rainwater per year that could be used from March to November without
\end{abstract}


deficit, covering the needs of vertical gardens in the area and adding availability for other uses. Finally, it is concluded that this type of systems allow mitigating the damages to the floodable infrastructure, reaching enormous social, landscape and ecological benefits.

Keywords: pluvial use, Flow Master, wetland, La Vaca Wetland, floods, Pipe Flow Expert, risk, Sustainable Drainage Systems.

\section{Resumo}

Os Sistemas Urbanos de Drenagem Sustentáveis (SUDS) na área de influência do pantanal La Vaca são uma alternativa para promover o uso eficiente do recurso hídrico. Para sua simulação se propõe três componentes principais: avaliação das principais áreas de captação de água da chuva, otimização das condições de operação hidráulica para um jardim vertical em Corabastos de acordo com a hidrologia e, por último, a simulação do sistema hidráulico proposto no software Pipe Flow Expert. Os resultados da simulação sugerem que implementar os SUDS sobre as coberturas de Corabastos são técnicas factíveis dado que não existe nenhum período de completa seca durante o ano na localidade de Kennedy; isso permitirá obter cerca de 1'537.000 litros de água da chuva anualmente, que poderia ser aproveitada de março a novembro sem déficit, cobriria as necessidades de jardins verticais na área e adicionaria disponibilidade para outros usos. Finalmente, se conclui que estes tipos de sistemas permitem mitigar os efeitos nàs infraestruturas inundáveis com enormes benefícios sociais, paisagísticos e ecológicos.

Palavras-chave: uso pluvial, Flow Master, pantanal, pantanal La Vaca, inundações, Pipe Flow Expert, risco, sistema de drenagem urbana sustentável 


\section{INTRODUCCIÓN}

En la actualidad mitigar el riesgo de inundación a partir de sistemas de drenaje sostenible requiere la inclusión de medidas estructurales que permitan controlar grandes volúmenes de escorrentía [1], [2]. En ese sentido considerar componentes naturales asociados a cubiertas vegetadas reduce el volumen de escorrentía, ya que aumenta la retención hídrica, la evapotranspiración e infiltración [3], [4]. En tanto la implementación de SUDS asociados con techos verdes, jardines verticales y humedales artificiales aumenta la capacidad del sistema urbano para gestionar los flujos de agua [5].

Colombia cuenta con 4 de las 214 grandes cuencas del mundo, correspondientes a los ríos Magdalena, Guaviare, Casanare y Meta. Si bien esta riqueza es enorme comparada con otros países, es necesario analizar la distribución de este recurso, donde contradictoriamente las zonas más pobladas del país son las más vulnerables a eventos de escasez [6]; es allí donde las alternativas de suministro hídrico en cualquiera de sus usos toma importancia.

Aprovechar las lluvias es una técnica ancestral donde el desarrollo de la humanidad siempre ha girado en torno al manejo del agua, los muiscas, por ejemplo, utilizaban técnicas de manejo de inundaciones, las cuales hoy en día son un indicador de que se deben mejorar los mecanismos de respuesta en el marco de gestión del riesgo [7]. Colombia es un país con una gran riqueza hídrica, estas condiciones ideales facilitan el acceso al recurso y el hecho de que no existen ciclos estacionales ha dejado de lado el desarrollo de tecnologías alternativas para el manejo de agua, entre ellas el aprovechamiento de agua lluvia [8].

Desde la época colonial en ciudades como Bogotá se ha dado un proceso de separación del paisaje causando un desequilibrio en la dinámica de los procesos naturales, es por eso que la adaptación al cambio climático exige un proceso de restauración y la incorporación de estrategias de ingeniería que en últimas fomenten la productividad, la biodiversidad y las relaciones culturales que se han degradado con el paso del tiempo [9]. Es allí, donde surgen los Sistemas Urbanos de Drenaje Sostenible (SUDS) como herramientas promotoras de la adecuada gestión del agua, dando respuesta al aumento de la impermeabilidad urbana, la pérdida de la intercepción natural del agua y la evapotranspiración; por otra parte, responden a problemas de calidad que atañen fundamentalmente a la contaminación de los medios receptores [10].
En Europa, la implementación de SUDS se ha utilizado como estrategia en el control de inundaciones. El uso de los SUDS se ha extendido en países del centro y norte de Europa. Por ejemplo, en Francia en el parque tecnológico Porte des Alpes en Lyon se integran cunetas verdes, drenes filtrantes, estanques de retención y depósitos de infiltración. En cambio, en Holanda los desarrollos de los SUDS se concentran en cubiertas vegetadas, pavimentos porosos y almacenamiento de aguas lluvias [11]. De igual manera en Latinoamérica la implementación de los SUDS se desarrolla con el fin de mitigar las inundaciones como es el caso de Chile que protege y amplía la infraestructura verde en el corredor ribereño del río Mapocho, para así mejorar la calidad del curso del agua, facilitar la infiltración del agua en el suelo, mitigar la erosión lateral y controlar las crecidas de dicho río [12].

En Colombia se inician algunos procesos de consultoría con el estudio de factibilidad técnica ambiental en el sistema de drenaje pluvial en el borde norte de la capital. No obstante, son pocos los SUDS implementados en la ciudad. Así pues, una de las grandes problemáticas de Bogotá es la amortiguación de aguas lluvias en el espacio público [13].

En la zona de estudio, el conflicto socio ecológico se remonta desde los inicios de urbanización de la localidad de Kennedy en la década de 1930 alrededor de la zona de influencia del humedal La Vaca (en adelante nombrado HLV), resaltando la creación de la central de abastos de alimentos más grande del país (Corabastos S.A.), con cerca de 420.000 $\mathrm{m}^{2}$. Con el paso del tiempo, los habitantes del sector han sido vulnerables en la época invernal ya que habitan en una zona que está catalogada con amenaza media - alta de inundación [14]. Producto de esta invasión, el HLV presenta disminución del área, dejando dos fragmentos (sector norte y sector sur) totalmente desconectados, colmatados, con poca área natural disponible, un pronunciado déficit hídrico y fuerte presión social, lo que significa una reducción sustancial de su capacidad de almacenamiento y amortiguamiento [15].

La presente investigación tiene como objetivo proponer nuevas alternativas para darle un uso eficiente al agua y mitigar las inundaciones a partir de la simulación hidrológica de un sistema para abastecer jardines verticales ubicados en la zona de influencia del HLV, todo lo anterior, asumiendo el gran potencial y los beneficios que tendría el desarrollo de SUDS para mejorar la sostenibilidad del recurso hídrico a la luz de la protección Ramsar con la que cuentan los humedales distritales. 
El presente artículo, realiza un análisis de como captar el agua de los techos en Corabastos, se verifica hidráulicamente la optimización de transporte en canaletas a tanques para posteriormente hacer un sistema de riego a presión para jardines verticales, utilizando agua lluvia para el suministro y software de simulación hidráulica.

\section{MATERIALES Y MÉTODOS}

El estudio se realizó bajo un enfoque cuantitativo, donde los objetivos se abordaron desde un alcance descriptivo y correlacional. La metodología constó de seis fases: recolección de información y diagnóstico territorial, selección del área de captación pluvial, análisis de disponibilidad pluvial, dimensionamiento del sistema de aprovechamiento, articulación con SUDS en el marco de crecimiento verde y pronóstico para el éxito de la propuesta.

\section{A. Área de estudio}

Inicialmente se partió de una contextualización espacial, donde se tuvieron en cuenta factores como la delimitación de la zona de influencia del HLV, con la utilización de cartografía específica de la zona (planos hidrosanitarios, planos arquitectónicos, información del PMA, entre otros), observaciones en campo (tipo de cubiertas, espacios aptos para el almacenamiento); para en últimas, establecer los lugares con requerimiento de suministro y así fijar las áreas de captación.

El HLV se encuentra ubicado en el sur occidente de Bogotá- Colombia, tiene un área de 7,96 hectáreas, la cual se encuentra fragmentada en dos partes: norte y sur. Limita al Noroccidente con la plaza de mercado Corabastos y la avenida Dagoberto Mejía, al sur limita con los barrios Olivo y Villa Nelly. La población limítrofe pertenece a la localidad de Kennedy, la cual excedió los 979,914 habitantes [16]. De hecho, esta situación aumenta la presión urbana sobre el HLV. En efecto se puede identificar que dicho humedal sufrió su más acelerada transformación en los últimos veinte años. Históricamente, el humedal ha disminuido aproximadamente el $88 \%$ de su área, cubriendo solo el $11 \%$ del área total [17], [18]. La vegetación del humedal de la Vaca predominante se caracteriza por la presencia de diferentes Juncos (Scirpus califormucus), Enena (Typa anutiotia) Polígono (Polygommi punctatum), Lengua de vaca (Rumex obtusifolius) y Duarte (fíidens laevis), entre otros [19].
Las visitas a campo consistieron en la identificación de la situación actual del humedal, caracterización de los muros y cubiertas más aptas para llevar a cabo el sistema de aprovechamiento de agua lluvia y recopilación de información por parte de los líderes comunitarios encargados de la administración del HLV y del Departamento de Infraestructura y Medio Ambiente de Corabastos.

\section{B. Datos hidrometereológicos y eventos de inundación}

Los datos de precipitación son obtenidos de la estación meteorológica de Kennedy, que forma parte de la red de monitoreo de calidad del aire de la Secretaria Distrital de Ambiente (SDA) en la ciudad de Bogotá, la cual aporta un marco integral para hacer pronósticos hidrometeorológicos.

Por otro lado, se elaboró un análisis de los eventos de inundaciones registrados en la base de datos del Idiger para tener en cuenta la magnitud y reiteración del riesgo; posteriormente, de acuerdo a los registros pluviométricos históricos de la estación meteorológica más cercana con un rango disponible de datos considerable, se examinó la precipitación media mensual. Una vez realizado lo anterior, se cuantificó la intensidad de lluvia por medio de las curvas IDF dispuestas por el departamento de ingeniería especializada del acueducto de Bogotá; para finalmente proceder a la simulación hidrológica del sistema de aprovechamiento empezando por el caudal de diseño, haciendo uso del método racional, mostrado a continuación:

\section{Caudal de diseño instantáneo para canaletas y promedio mensual para tanques de almacenamiento}

Según la norma NS 085 del acueducto de Bogotá el método racional consta de:

$$
Q=C I A
$$

Donde,

I= Intensidad de la lluvia en litro/segundo*Hectárea. $A=$ Área de drenaje en Hectáreas.

$\mathrm{C}=$ Coeficiente de escorrentía ( 0,85 para cubiertas). $\mathrm{Q}=$ Caudal en litros/segundo.

$$
\text { INTENSIDAD }=C_{1}\left(D U R A C I O ́ N+X_{0}\right)^{C_{2}}
$$


Haciendo uso de los coeficientes proporcionados por el acueducto en la tabla 1 se halló la intensidad y se operó con el área de las cubiertas calculada por medio de SIG, para obtener el respectivo caudal de diseño. Es necesario aclarar, que para hallar el caudal de diseño de las canaletas que captan el agua lluvia de los techos, se utiliza el instantáneo anteriormente mencionado de las curvas IDF del acueducto de Bogotá.

Es diferente para hacer el dimensionamiento de los tanques de almacenamiento, ya que este se realizó mediante un análisis de oferta y demanda, se utilizó el método racional pero con los datos de precipitación promedio anual, teniendo en cuenta el consumo de los techos verdes, a partir de datos históricos de más de 10 años de la estación del Ideam; se analizaron las cantidades de agua contempladas por mes para obtener el volumen del tanque de almacenamiento, asegurando que tenga la capacidad para almacenar los meses de máxima precipitación.

\section{Dimensionamiento de canaletas para captar el agua lluvia de las cubiertas}

Este apartado se desarrolló partiendo de que la capacidad de conducción de agua de un canal, depende de la geometría de su sección transversal, sus dimensiones, la pendiente del canal y los materiales de elaboración, de los que dependen las perdidas por fricción [20]. Cuando ya se tiene por el método racional el caudal instantáneo de las curvas IDF, se procede al dimensionamiento de las estructuras de conducción de agua lluvia aportada por las cubiertas anteriormente seleccionadas, para lo cual se hizo uso de la fórmula de Manning (ecuación 3) para canales que van a superficie libre, que se relaciona a continuación:

Donde:

$$
V=\left(\frac{1}{n R}\right)^{2 / 3} S^{1 / 2}
$$

R: Radio hidráulico en metros, para canales con sección circular $=\mathrm{D} / 4$.

n: Coeficiente de rugosidad de Manning según el material.0.013 pvc (Este valor simula el envejecimiento del material, ya que con el tiempo la rugosidad aumenta).

S: Pendiente del canal en metro/metro.

D: Diámetro en metros

Q: $V^{*} A$
3. Selección de la bomba para el transporte de agua lluvia almacenada para el riego de los jardines verticales

Para la selección de la bomba se tuvo en cuenta las pérdidas a las que está sujeto el sistema, con la ecuación de Darcy-Weisbach que se relaciona a continuación, que considera la pérdida de carga por fricción (ecuación 4) de la misma manera, se selecciona el mejor diámetro de tubería para optimizar el uso energético de acuerdo a la velocidad:

$$
h f=f \times \frac{L}{D} \times \frac{v^{2}}{2 g}
$$

Donde:

hf: es la pérdida de carga debida a la fricción, en m. f: factor de fricción de Darcy, adimensional.

L/D: relación entre la longitud y el diámetro de la tubería en $\mathrm{m}$.

$\mathrm{v}$ : velocidad media de flujo en $\mathrm{m} / \mathrm{s}$.

g: corresponde a la aceleración debida a la gravedad, y se supone constante $\left(9.81 \mathrm{~m} / \mathrm{s}^{2}\right)$.

\section{Análisis de oferta de agua lluvia según los datos de precipitación promedio anual contra la demanda hídrica de las especies seleccionadas para el jardín vertical y su densidad de siembra}

Con el propósito de contemplar el aprovechamiento del agua lluvia, se tomó toda la información anterior para realizar un análisis de oferta y demanda mensual asociado al abastecimiento de tres estructuras verdes (jardines verticales) ubicados estratégicamente alrededor del humedal como parte del complejo ecológico de la zona.

Se tomó como referencia la norma técnica NS 085 que muestra los criterios de diseño con la utilización del método racional, además de un dato de requerimiento hídrico para las plantas, fijado en $21 \mathrm{~mm}$ por planta a la semana, dato que surge tras la realización de ensayos experimentales realizados por la administración del HLV. De esta forma fue posible dimensionar los tanques de almacenamiento de agua lluvia a la medida, según la lluvia y la demanda por el jardín vertical. 
Finalmente, se realiza una simulación del sistema de riego de agua lluvia a presión por medio de sistemas de bombeo de acuerdo a: espacio disponible, necesidad de cumplir funciones ecológicas, atenuación de caudales para prevenir inundaciones y uso del agua.

Para tal fin se utilizó el software Flow Master de Bentley para definir las necesidades de bombeo en términos de metros de columna de agua y el software PipeFlow Expert para verificar el comportamiento del sistema completo con las respectivas pérdidas por fricción y accesorios.

\section{RESULTADOS Y DISCUSIÓN}

\section{A. Selección de áreas de captación}

Después de realizar visitas de campo, análisis hidrosanitario y análisis espacial en SIG, se seleccionaron los SUDS más adecuados teniendo en cuenta que la zona de influencia directa del humedal se compone de la infraestructura de Corabastos que presenta un escenario uniforme y con factibilidad de intervenir, de esta forma se planteó un Sistema de Captación de Agua Lluvia en Techos (Scapt), en el que se prioriza el suministro de riego a jardines verticales ubicados en las proximidades del humedal e incorpora lavado de pisos, agua para descarga de sanitarios, fachadas y alimentación del caudal del HLV. Por otro lado, se contemplaron las cubiertas vegetales en un estudio preliminar para techos verdes aplicado al resto de bodegas con miras a atenuar la escorrentía de la zona y obtener beneficios ambientales, todo lo anterior en el marco del crecimiento verde.

Las cubiertas seleccionadas se muestran en la figura 6 por su proximidad a los jardines verticales que hacen parte del desarrollo teórico-práctico de [18], con un área de $1633,65 \mathrm{~m}^{2}$ y $3382,30 \mathrm{~m}^{2}$ respectivamente, dichas cubiertas son planas con pendiente dirigida al HLV, a diferencia de la mayoría de bodegas que son de tipo mixto (planas y a dos aguas) como se muestra en la figura 1.

Las cubiertas que no colindan con el HLV, fueron descartadas dado que no existe conectividad entre las bodegas para hacer llegar el agua a la zona de suministro que bordea el humedal. Las aguas serán conducidas a un sistema de almacenamiento y por medio de un sistema de bombeo se hará llegar hacia las zonas de los jardines verticales, para ello a continuación se abordará el análisis de la tendencia de la precipitación de la zona.

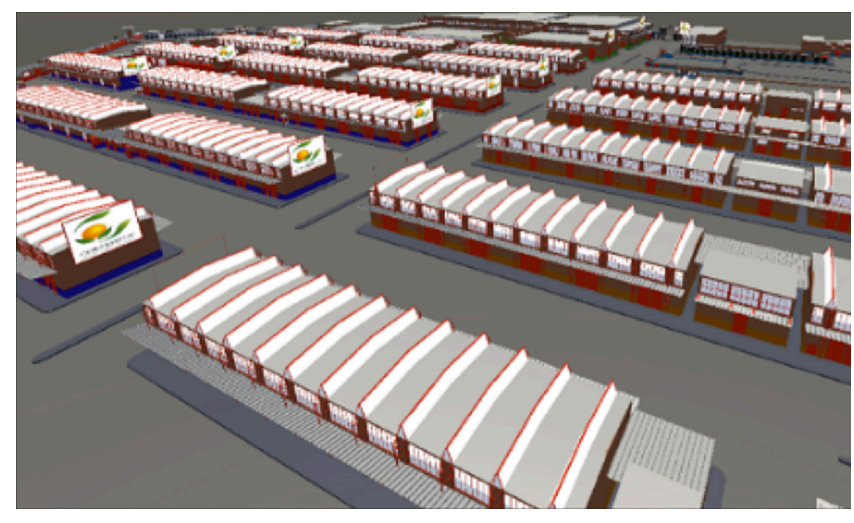

Figura 1. Panorámica de las cubiertas de las bodegas de Corabastos.

Fuente: visor virtual de Corabastos, 2019.

\section{B. Análisis de la precipitación promedio anual}

Al analizar la figura 2 la distribución de la precipitación se muestra de tipo bimodal con dos periodos húmedos alternados con dos periodos secos. Las mayores precipitaciones ocurren entre abril y mayo, y de septiembre a noviembre. Mientras que los periodos más secos se presentan de diciembre a marzo y de junio a agosto.

A partir del análisis de la tendencia de precipitación en la zona de influencia del HLV se deduce que es factible considerar un sistema de aprovechamiento de agua lluvia dado que no existe ningún periodo de completa sequia durante todo el año, sin embargo, se debe prever que para los meses como diciembre, enero, febrero, julio y agosto el recurso estará disponible en menor cantidad.

En la figura 3 se asocian los registros de inundaciones del Idiger desde el año 1921 al año 2018 en la localidad de Kennedy [21], se evidencia que el riesgo ha sido constante hasta la actualidad; posiblemente debido a la poca planeación con la que se organizaron estos barrios y la forma en que se asentaron ilegalmente en la zona. Se resaltan barrios como Patio Bonito y Tintalito que están localizados justo en las proximidades del HLV y presentan eventos de inundaciones continuos desde 1921 hasta la actualidad. 


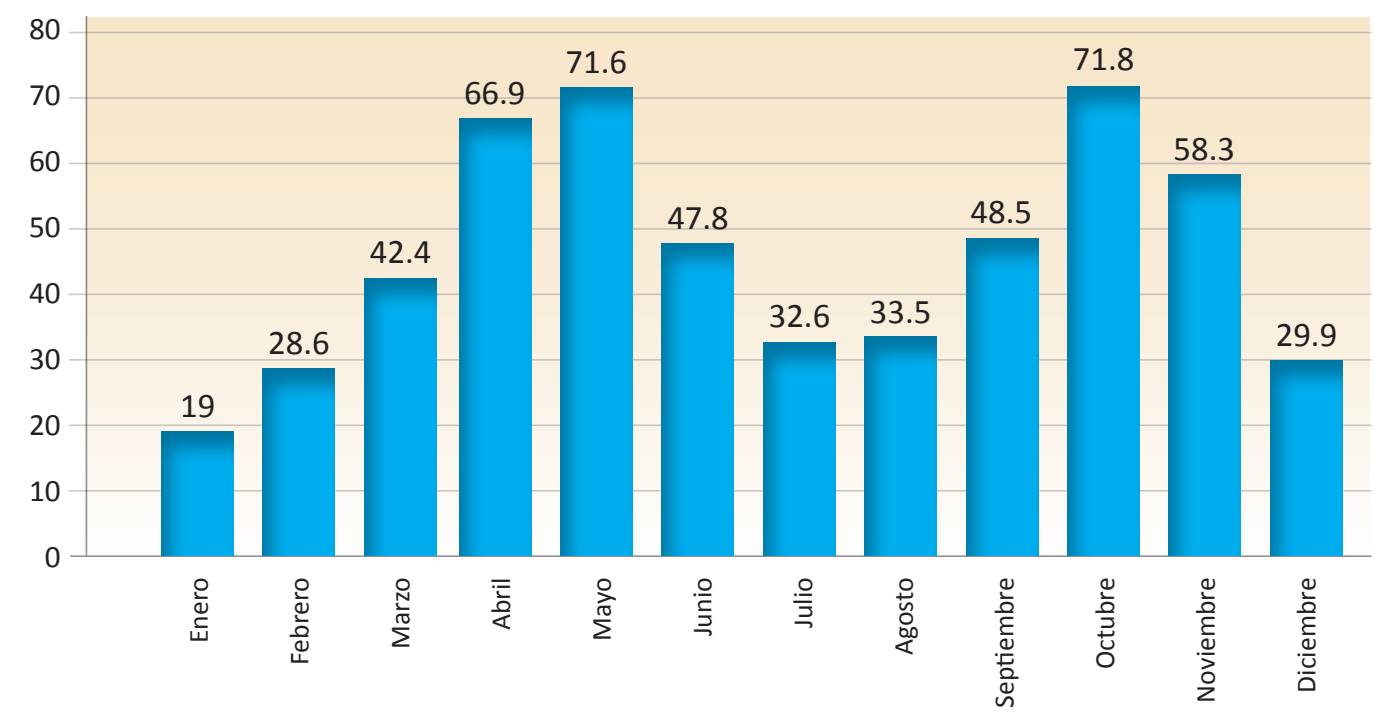

Fig. 2. Precipitación media mensual en mm de la zona HLV.

Fuente: estación pluviométrica Bosa Barreno P-051.

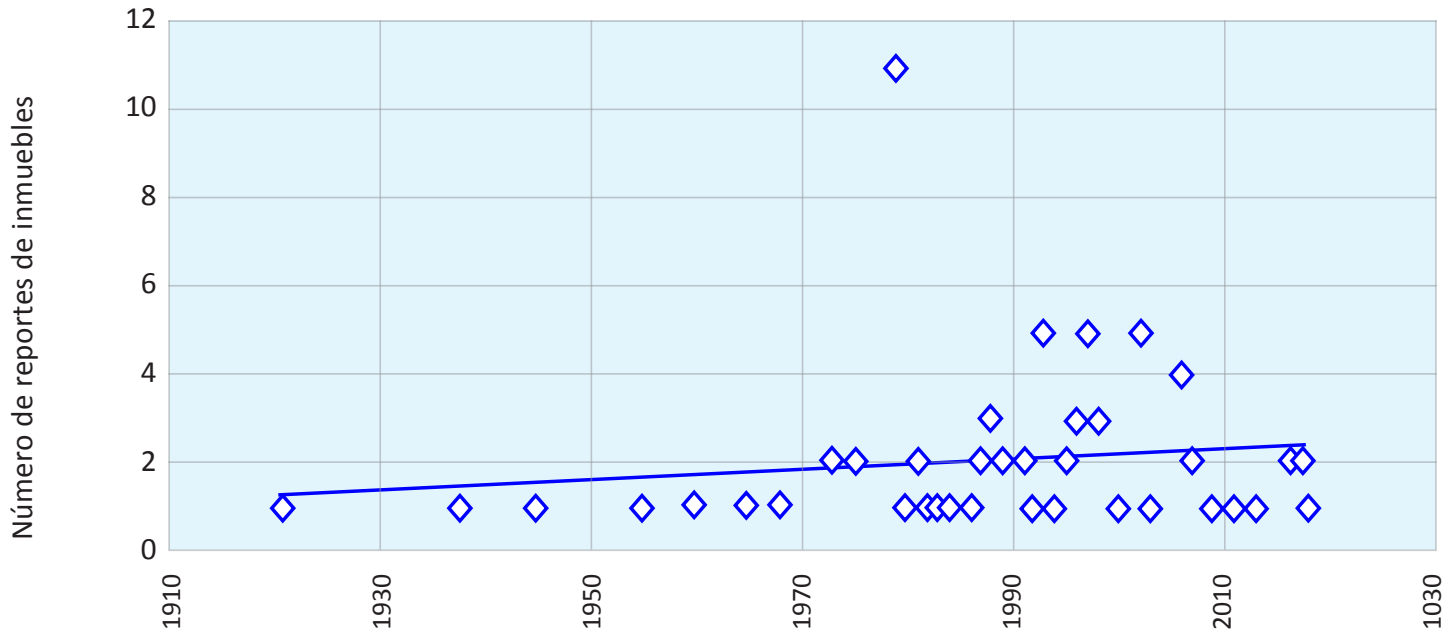

Figura 3. Registros históricos de eventos de inundación en la localidad de Kennedy entre los años 1921-2018.

Fuente: adaptado de base de datos del Idiger.

En Bogotá el agua fluye desde los cerros orientales por quebradas, ríos y humedales hasta el río Bogotá, atravesando de oriente a occidente la ciudad [22]. Es decir, que si se intercepta y aprovecha el agua lluvia justamente en la zona donde se ubica
Corabastos se mitiga de manera inmediata el porcentaje de escorrentía que amenaza a los barrios bajos de la localidad que se encuentran en amenaza alta de inundación en cercanías al río Bogotá (figura 4). 


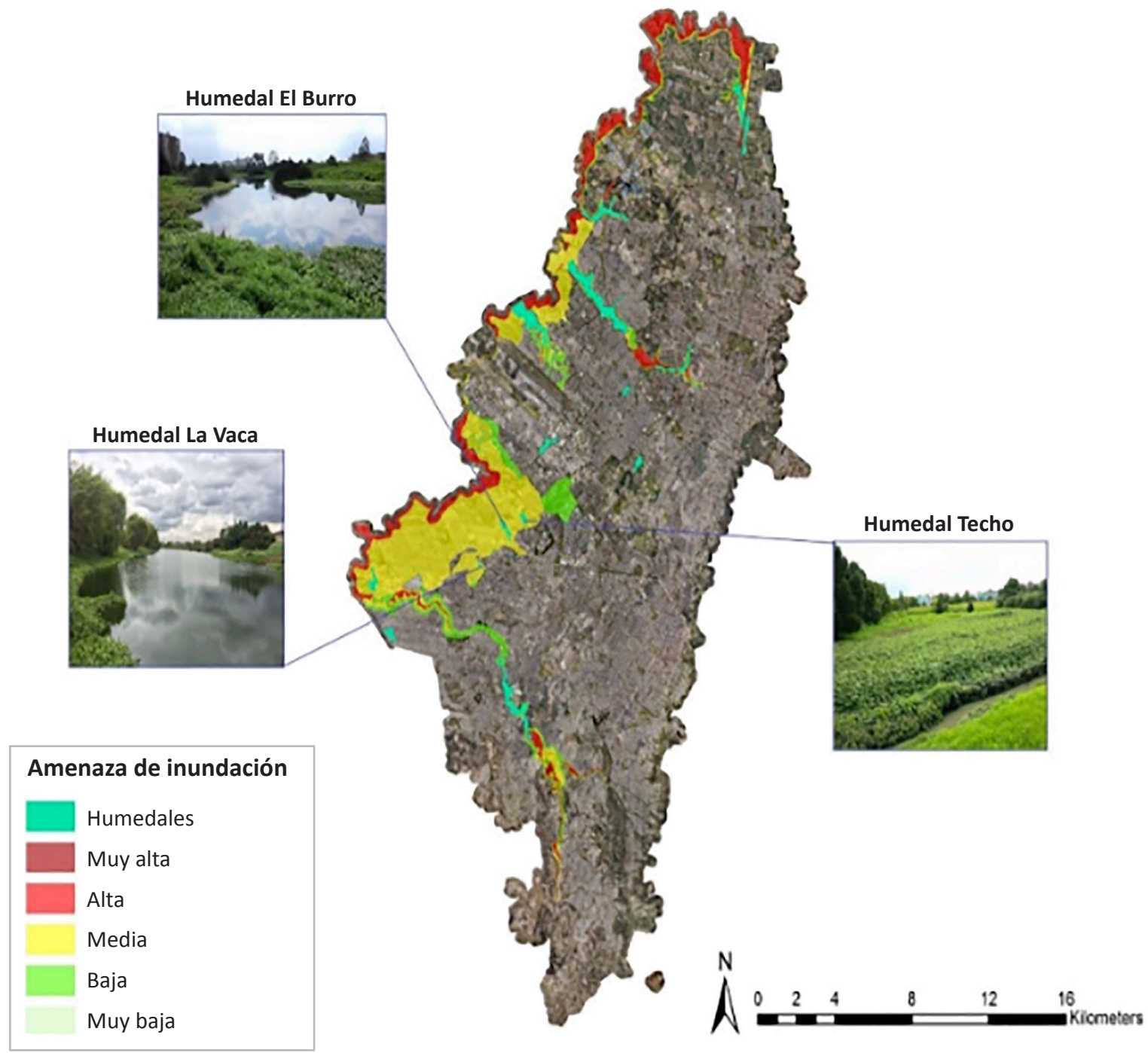

Fig. 4. Amenaza de inundación en Bogotá.

Fuente: elaboración propia, adaptado de Ideca, 2018.

Teniendo en cuenta lo anterior, se procedió a delimitar la microcuenca asociada al humedal La Vaca a partir de un modelo de elevación digital (DEM), para ello se utilizó la herramienta "Hidrology" del software ArcGis se identificó la acumulación y dirección de los flujos en la zona, como se observa en la figura 5 , donde se puede deducir que los flujos justamente tienden hacia el río Bogotá y las principales acumulaciones se observan en la zona del humedal, en sus sectores norte y sur.

Como se puede evidenciar en las gráficas anteriores, la acumulación de flujo de agua que da la herramienta SIG, a partir de un modelo de elevación digital de terreno descargado del Servicio Geológico de Estados Unidos, coincide con los datos de amenaza de inundación de Ideca. Esto se debe a que el cambio de uso de suelo de terreno natural a construido, hace que la infiltración sea mínima por tanto aumenta la cantidad de agua superficial debido a la impermeabilización ocasionada por los sistemas constructivos de la ciudad a lo largo del tiempo, lo que aumenta la amenaza de inundación.

He allí la importancia de la restauración ecológica en esta zona, de manera que se logre maximizar la capacidad del humedal para amortiguar y retener estas grandes cantidades de agua. 

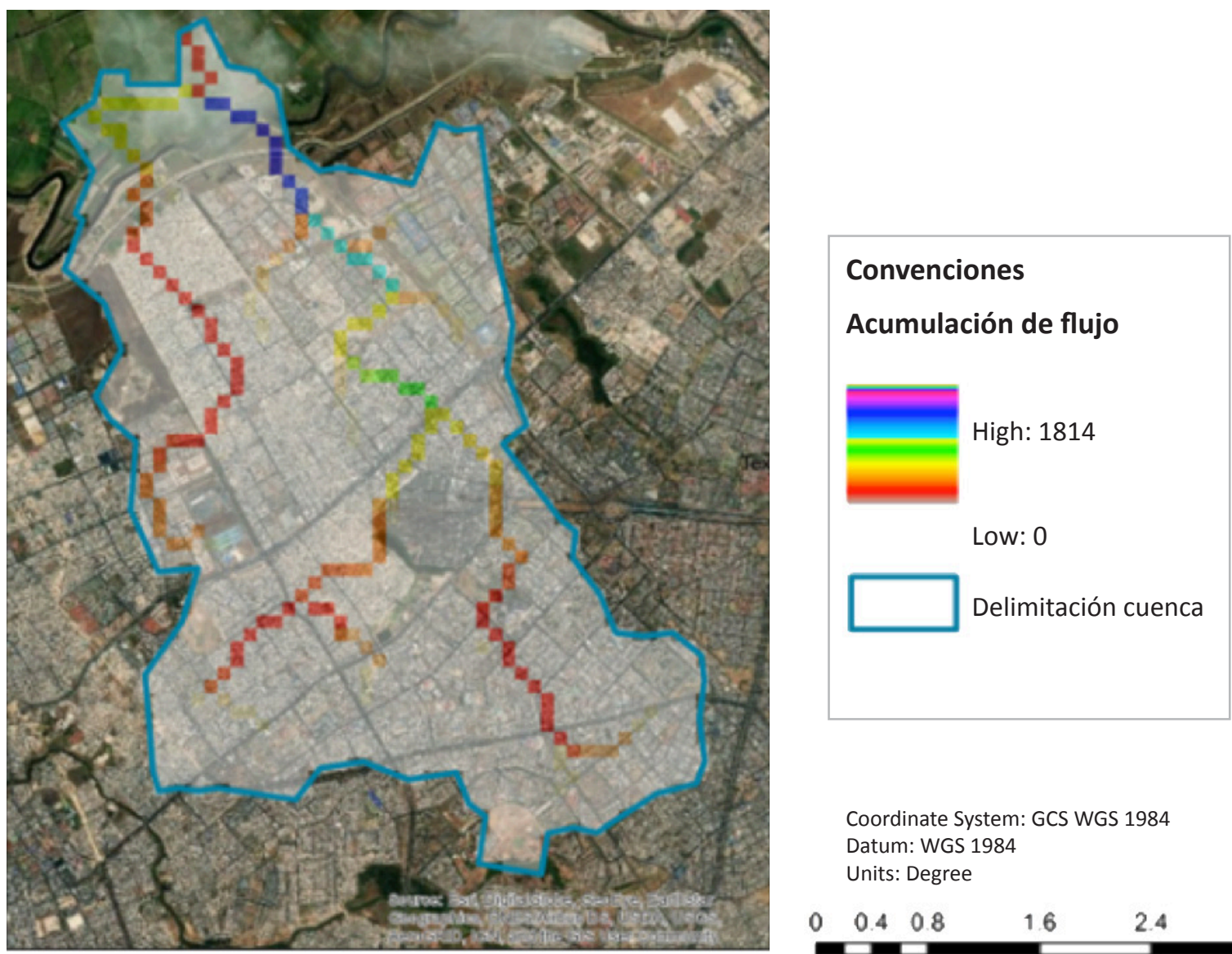

Fig. 5. Acumulación del flujo hídrico en la microcuenca del HLV. Fuente: elaboración propia, adaptado de Ideca, 2018.

Con la finalidad de no sobreestimar un aguacero poco usual, se utiliza un periodo de retorno de 3 años (tabla 1). Con el fin de realizar el cálculo de las estructuras hidráulicas de drenaje de las cubiertas de manera que soporten un aguacero de esta magnitud, a partir del caudal de diseño, que es la relación de la intensidad de lluvia en la zona según los datos del acueducto de Bogotá, relacionados por el método racional (ver tabla 2).

\begin{tabular}{|c|c|c|}
\hline \multicolumn{3}{|c|}{ Tiempo de retorno 3 años } \\
\hline C1 & Xo & C2 \\
\hline Monóxido de Carbono & $<10$ ppm & Xo \\
\hline 2093.42 & 20.6 & -1.01687 \\
\hline
\end{tabular}

Tabla 1. Coeficientes ecuación idf para las coordenadas: 4³7'52”N 749'34”'W (HLV). Fuente: equipo de ingeniería especializada del acueducto de Bogotá.

\begin{tabular}{|c|c|c|}
\hline Sistema & Intensidad $(\mathrm{L} / \mathrm{s}$ ha) & Intensidad $(\mathrm{L} / \mathrm{s})$ \\
\hline Cubierta 1 & 197,9225 & 27,4726 \\
\hline Cubierta 2 & 190,2645 & 54,7115 \\
\hline
\end{tabular}

Tabla 2. Intensidad y caudal de diseño a partir del método racional. Fuente: elaboración propia.

\section{Análisis de oferta de agua lluvia según los datos de precipitación promedio anual contra la demanda hídrica de las especies seleccionadas para el jardín vertical y su densidad de siembra}

Los tres jardines verticales de la zona, debido a su ubicación estratégica con respecto al humedal, tienen las mismas dimensiones, un área de 43,2 $\mathrm{m}^{2}$ 
cada uno. Cada jardín se compone de ocho plantas (enredaderas) de las especies: Uncaria guianensis conocida como uña de gato y Fagonia cretica conocida como manto de María. El requerimiento hídrico se fijó en $21 \mathrm{~mm}$ por planta a la semana y esto se sumó a los otros usos mencionados con anterioridad para consolidar la tabla 1 de Análisis de oferta y demanda hídrica.
Las necesidades de agua por cada jardín se obtuvieron considerando un área de riego con un ancho de 1,2 metros. Al realizar el cálculo, se verificó que la demanda para otros usos, tendrá un volumen disponible adicional al riego para actividades como limpieza, sanitarios, orinales, lavado de fachadas y pisos, entre otros en ciertos meses del año (ver tabla 3).

\begin{tabular}{|c|c|c|c|c|c|c|c|c|}
\hline 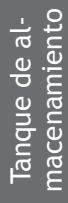 & $\stackrel{\text { }}{\Sigma}$ & 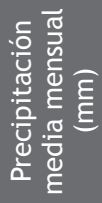 & 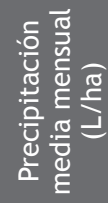 & 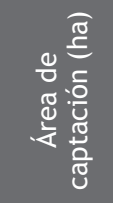 & 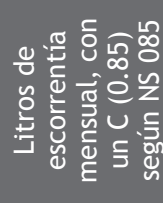 & 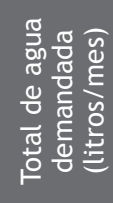 & 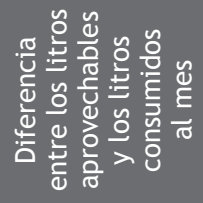 & 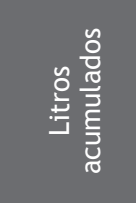 \\
\hline \multirow{12}{*}{1} & ENE & & 190000 & \multirow{12}{*}{0.16337} & 26383.45 & \multirow{12}{*}{41919.2} & -15535.75 & \multirow{2}{*}{-18140.91} \\
\hline & FEB & & 286000 & & 39714.03 & & -2205.17 & \\
\hline & MAR & 42.4 & 424000 & & 58876.75 & & 16957.55 & \multirow{4}{*}{149896.59} \\
\hline & $\mathrm{ABR}$ & 66.9 & 669000 & & 92897.51 & & 50978.31 & \\
\hline & MAY & 71.6 & 716000 & & 99423.94 & & 57504.74 & \\
\hline & JUN & 47.8 & 478000 & & 66375.2 & & 24456 & \\
\hline & JUL & 32.6 & 326000 & & 45268.44 & & 3349.24 & \multirow{2}{*}{7948.23} \\
\hline & AGO & 33.5 & 335000 & & 46518.18 & & 4598.98 & \\
\hline & SEP & 48.5 & 485000 & & 67347.22 & & 25428.02 & \multirow{6}{*}{-35819.79} \\
\hline & OCT & 71.8 & 718000 & & 99701.66 & & 57782.46 & \\
\hline & NOV & 58.3 & 583000 & & 80955.53 & & 39036.33 & \\
\hline & DIC & & 299000 & & 41519.21 & & -399.99 & \\
\hline \multirow{12}{*}{2} & ENE & 19 & 190000 & \multirow{12}{*}{0.33823} & 54624.15 & \multirow{12}{*}{86209.6} & -31585.46 & \\
\hline & FEB & 28.6 & 286000 & & 82223.71 & & -3985.89 & \\
\hline & MAR & 42.4 & 424000 & & 121898.1 & & 35688.49 & \\
\hline & $\mathrm{ABR}$ & 66.9 & 669000 & & 192334.5 & & 106124.89 & 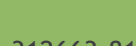 \\
\hline & MAY & 71.6 & 716000 & & 205846.8 & & 119637.18 & \\
\hline & JUN & 47.8 & 478000 & & 137422.9 & & 51213.25 & \\
\hline & JUL & 32.6 & 326000 & & 93723.53 & & 7513.93 & 7 \\
\hline & AGO & 33.5 & 335000 & & 96310.99 & & 10101.39 & \\
\hline & SEP & 48.5 & 485000 & & 139435.3 & & 53225.72 & \\
\hline & ОСТ & 71.8 & 718000 & & 206421.8 & & 120212.17 & 254838.16 \\
\hline & NOV & 58.3 & 583000 & & 167609.9 & & 81400.28 & \\
\hline & DIC & 29.9 & 299000 & & 85961.15 & & -248.45 & \\
\hline
\end{tabular}


Al analizar la tabla 3, para el caso de la cubierta 1, se tendría un déficit total de $18,5 \mathrm{~m}^{3}$ acumulados en los meses de diciembre, enero y febrero; y por otro lado, para el caso de la cubierta 2, se tendría un déficit acumulado de $45,9 \mathrm{~m}^{3}$ para los mismos meses. Sin embargo, dicho déficit se compensa con el acumulado de los meses anteriores que, al ser lluviosos (señalados en verde), presentan ofertas hídricas muy grandes. Esto muestra que el sistema sería aplicable y funcional; siempre y cuando al agua almacenada se le dé un monitoreo y manejo en términos de su calidad.

Por otro lado, con estos datos se dimensionaron los tanques de almacenamiento y se obtuvo una capacidad de $60 \mathrm{~m}^{3}$ y $120 \mathrm{~m}^{3}$ respectivamente.

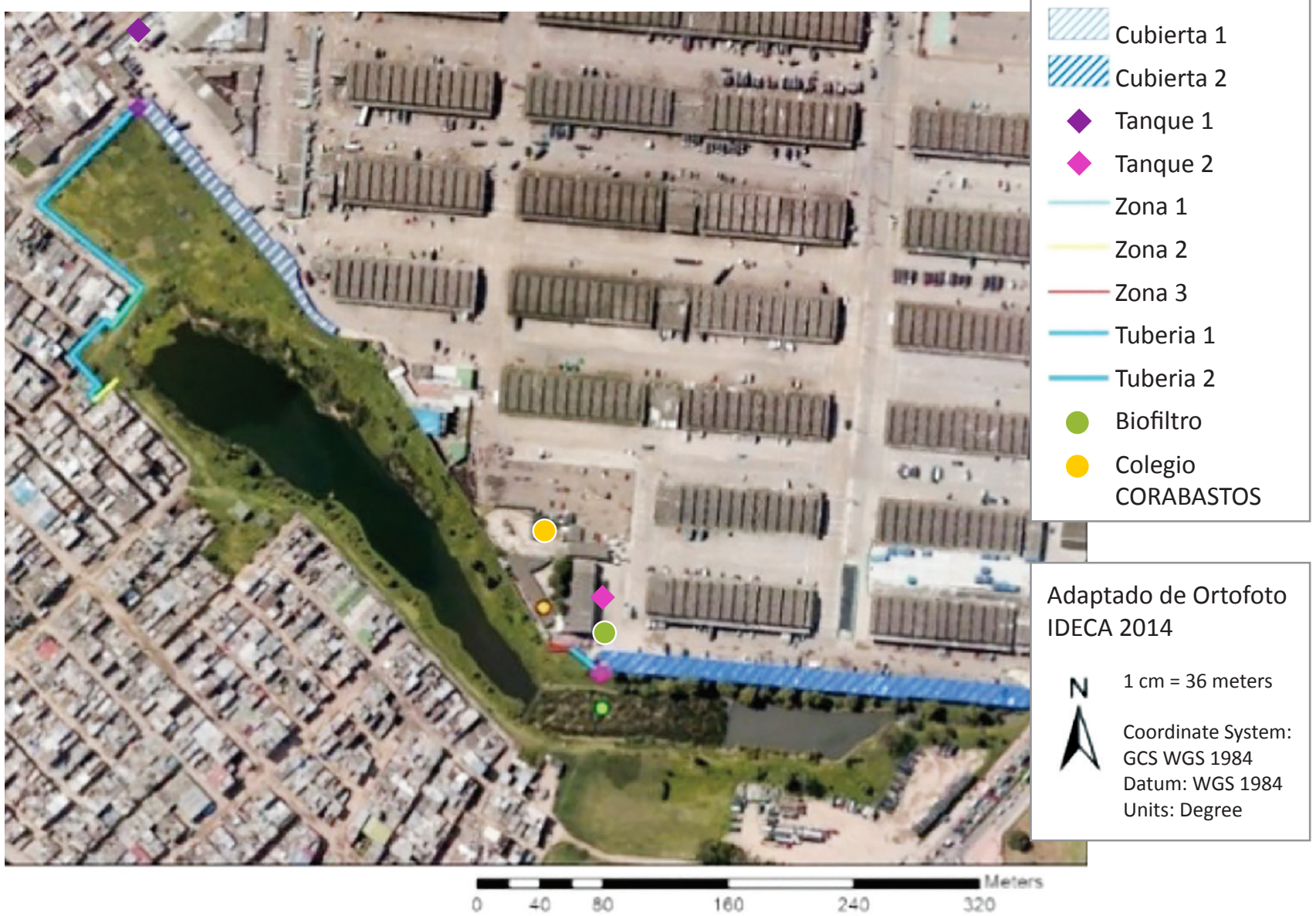

Fig. 6. Sistema de captación, conducción y almacenamiento de agua lluvia en el HLV. Fuente: elaboración propia.

A partir de la ecuación de Dary-Weisbach, ecuación utilizada para la simulación de sistemas de agua a presión (tabla 4) se cuantificaron las pérdidas ocasionadas por fricción, por accesorios y por alturas, dato con el que se determinó la bomba requerida, se tuvo cuidado para que la velocidad media del agua al interior de la tubería no pasara de $2 \mathrm{~m} / \mathrm{s}$.

\begin{tabular}{|l|c|c|c|c|c|}
\hline Tubería & $\begin{array}{c}\text { Longitud } \\
\text { Total } \\
(\mathrm{m})\end{array}$ & $\begin{array}{c}\text { Diámetro } \\
(\mathrm{m})\end{array}$ & $\begin{array}{c}\text { Área } \\
\left(\mathrm{m}^{2}\right)\end{array}$ & $\begin{array}{c}\text { Velocidad } \\
(\mathrm{m} / \mathrm{s})\end{array}$ & $\begin{array}{c}\text { Pérdidas } \\
(\mathrm{m})\end{array}$ \\
\hline Tub. 1 & 301.6 & 0.0508 & 0.002 & 1.73 & 20.89 \\
\hline Tub. 2 & 51.72 & 0.0508 & 0.002 & 1.3815 & 6.56 \\
\hline
\end{tabular}

Tabla 4. Características de las tuberías.

Fuente: elaboración propia. 
La idea de realizar la simulación es asegurar velocidades entre 0,6 y $2 \mathrm{~m} / \mathrm{s}$ para hacer que las pérdidas por fricción y por accesorios estén en rangos aceptables y así el costo del sistema de bombeo y de su operación sea el óptimo, lo anterior se cumple como se puede observar en la tabla anterior, de manera que ya se tendrían los diámetros a utilizar en el sistema de riego a presión.

En ese orden de ideas, también se realizó un cálculo preliminar (tabla 5) mediante el programa Flow Master de Bentley, con la finalidad de verificar las necesidades de sistemas de bombeo para los diferentes arreglos, lo cual nos indica que se necesita una presión de 4.95 metros de columna de agua en pérdidas por fricción para la tubería 1 y de 6.56 metros de columna de agua para el sistema, sin tener en cuenta las pérdidas menores que se deben a los accesorios, codos, tes, uniones entre otros accesorios necesarios en la instalación de sistemas hidráulicos lo cual se tendrá en cuenta en una simulación con el programa Pipe Flow Expert.

\begin{tabular}{|c|c|c|c|}
\hline Elevación 1 & $4.95 \mathrm{~m}$ & $\begin{array}{c}\text { Grado } \\
\text { energético } 2\end{array}$ & $3.65 \mathrm{~m}$ \\
\hline Elevación 2 & $3.60 \mathrm{~m}$ & $\begin{array}{c}\text { Grado } \\
\text { hidráulico } 1\end{array}$ & $4.95 \mathrm{~m}$ \\
\hline Longitud & 301.6 m & $\begin{array}{c}\text { Grado } \\
\text { hidráulico } 2\end{array}$ & $3.60 \mathrm{~m}$ \\
\hline $\begin{array}{l}\text { Altura de } \\
\text { rugosidad }\end{array}$ & $\begin{array}{c}0.0015 \\
\mathrm{~mm}\end{array}$ & Área de flujo & $0.3 \mathrm{ft}^{2}$ \\
\hline Diámetro & $0.193 \mathrm{~m}$ & $\begin{array}{l}\text { Perímetro } \\
\text { mojado }\end{array}$ & $0.6 \mathrm{~m}$ \\
\hline Descarga & $30 \mathrm{~L} / \mathrm{s}$ & Velocidad & $3.36 \mathrm{ft} / \mathrm{s}$ \\
\hline $\begin{array}{l}\text { Viscosidad } \\
\text { cinemática }\end{array}$ & $\begin{array}{l}1.140 \mathrm{e}- \\
006 \mathrm{~m}^{3} / \mathrm{s}\end{array}$ & $\begin{array}{l}\text { Velocidad de la } \\
\text { cabeza }\end{array}$ & $0.05 \mathrm{~m}$ \\
\hline $\begin{array}{c}\text { Peso } \\
\text { específico }\end{array}$ & $\begin{array}{c}9,810 \mathrm{~N} / \\
\mathrm{m}^{3}\end{array}$ & $\begin{array}{l}\text { Pendiente de } \\
\text { fricción }\end{array}$ & $0.004 \mathrm{ft} / \mathrm{ft}$ \\
\hline $\begin{array}{l}\text { Pérdida de la } \\
\text { cabeza }\end{array}$ & $4.44 \mathrm{ft}$ & $\begin{array}{l}\text { Factor de } \\
\text { fricción }\end{array}$ & 0.016 \\
\hline $\begin{array}{c}\text { Grado } \\
\text { energético } 1\end{array}$ & $5.01 \mathrm{~m}$ & $\begin{array}{l}\text { Numero de } \\
\text { Reynolds }\end{array}$ & $173,607.8$ \\
\hline
\end{tabular}

Tabla 5. Simulación en flow master para perdidas por fricción. Fuente: elaboración propia.

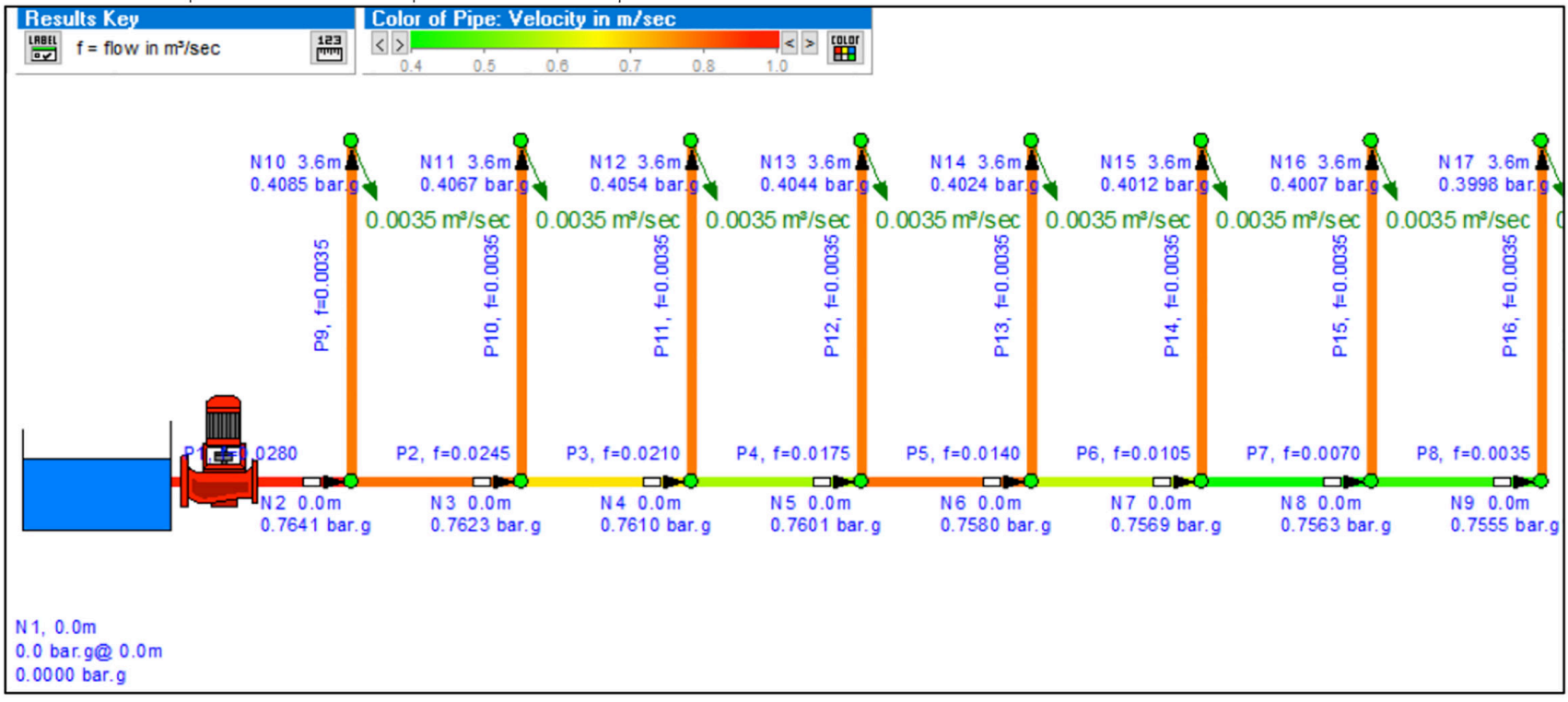

Fig. 7. Simulación en Pipe Flow Expert para el sistema completo.

Fuente: elaboración propia.

Por otro lado, se realizó una simulación de un tanque elevado mediante el software Pipe Flow Expert (figura 7) con la finalidad de verificar el comportamiento del sistema de tuberías a presión, con el sistema 1, de forma que la descarga sea de 3.5 litros por segundo en cada una de las salidas con una presión de 4.8 metros de columna de agua o 0.3998 Bar, presión suficiente para el abastecimiento de las plantas, lo que asegura el caudal requerido, vence las perdidas por fricción y accesorios como se puede observar en el siguiente gráfico. La bomba seleccionada es comercial, de IHM GS 150-10TW Referencia: 63980000A1 3500 RPM, 220/440 V, $10 \mathrm{HP}$, cuya curva característica se añadió al programa Pipe Flow Expert para realizar la simulación. 
Lo que indica la curva característica de la bomba (figura 8) y la simulación de Pipe Flow expert es que la bomba seleccionada se ajusta a las necesidades de agua del sistema requerido. Es de suma importancia realizar un adecuado diseño hidráulico que asegure que los caudales, presiones requeridas y energía requerida por el sistema sean óptimas.
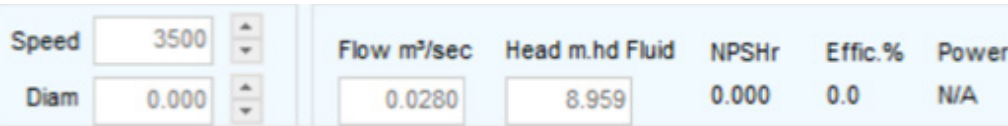

Calculate

Preferred Operating region between 0.0000 and $0.0000 \mathrm{~m}^{3} / \mathrm{sec}$

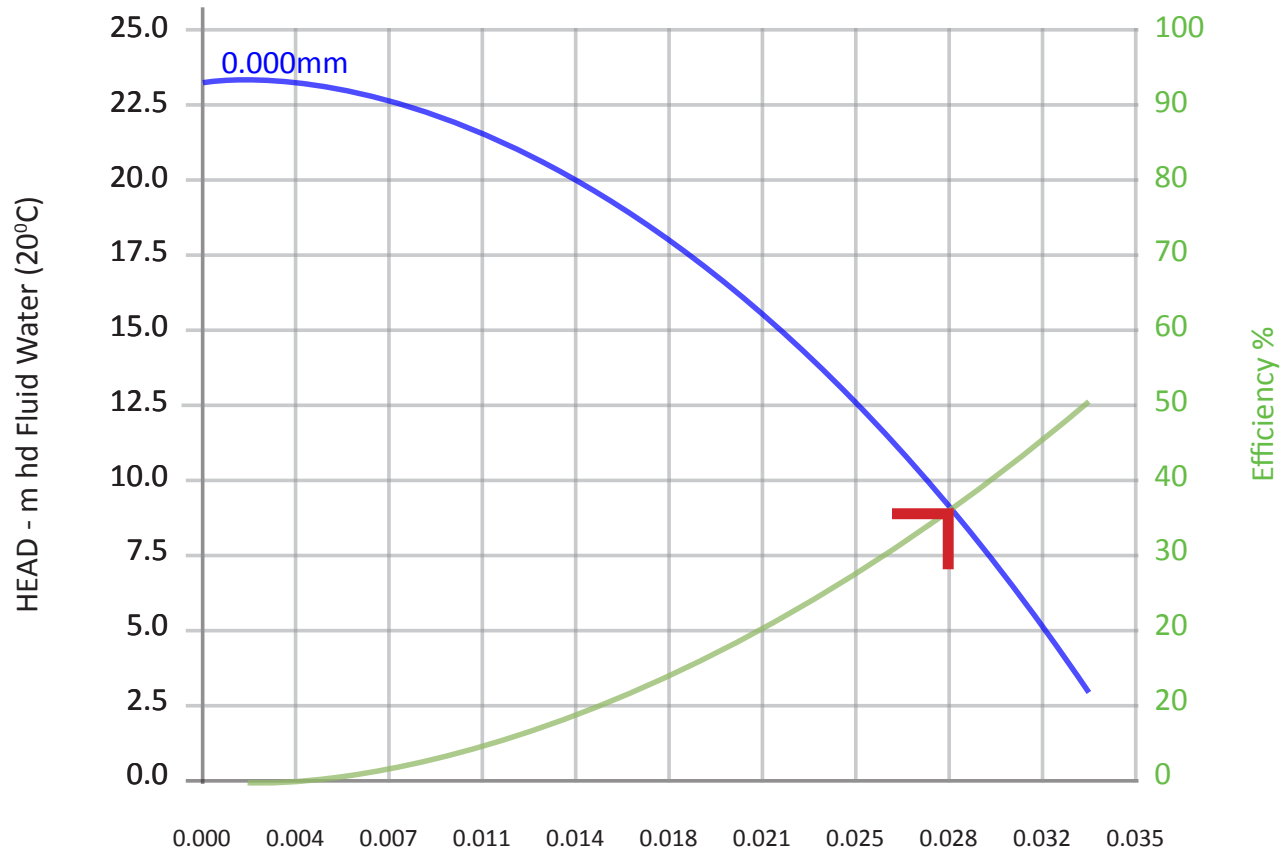

Fig. 8. Curva característica de la bomba seleccionada en el programa Pipe Flow Expert.

Fuente: elaboración propia.

Las tuberías por su parte, fueron dispuestas a la par de la estructura que delimita el humedal (figura 7) de manera que no se vean interceptados los senderos por donde transitan generalmente grupos de personas en actividades asociadas a la educación ambiental y a la administración del humedal en general.

Por otro lado, para el dimensionamiento del sistema de captación se seleccionó el canal de tipo amazona de Pavco cuya relación se plantea en la tabla 6 con la fórmula de Manning.

\begin{tabular}{|l|c|c|}
\hline \multicolumn{1}{|c|}{ Criterio } & Cubierta 1 & Cubierta 2 \\
\hline Caudal de diseño (L/s) & 27.4726 & 54.7115 \\
\hline Diámetro $(\mathrm{m})$ & 0.254 & 0.254 \\
\hline $\mathrm{n}(\mathrm{PVC})$ & 0.013 & 0.013 \\
\hline Pendiente $(\mathrm{m} / \mathrm{m})$ & 0.002 & 0.008 \\
\hline Velocidad $(\mathrm{m} / \mathrm{s})$ & 0.5475 & 1.0951 \\
\hline Área $(\mathrm{m} 2)$ & 0.0507 & 0.0507 \\
\hline Caudal de corroboración $(\mathrm{L} / \mathrm{s})$ & 27.7446 & 55.4893 \\
\hline
\end{tabular}

Tabla 6. Características de las canaletas Fuente: Elaboración propia. 
De esta manera, se verifica por medio de la fórmula de Manning, que el caudal requerido, es posible ser llevado por una canaleta de 0.254 metros. De la misma manera, el estudio presenta los costos asociados al mejoramiento propuesto, de forma que se pueda presentar formalmente ante las autoridades correspondientes. En cuanto al estudio preliminar de los costos asociados al sistema de aprovechamiento propuesto y sus posibles beneficios monetarios, se relacionan en la tabla 7 en pesos colombianos al año 2019 de acuerdo a la lista de precios de Pavco y el portal CYPE Ingenieros S, A.:

\begin{tabular}{|c|c|c|c|}
\hline Descriptor & Cant. & $\begin{array}{c}\text { Valor } \\
\text { unitario (\$) }\end{array}$ & $\begin{array}{l}\text { Valor total } \\
(\$)\end{array}$ \\
\hline Tubería 2" & $209.12 \mathrm{~m}$ & 11,900 & $2,488,528$ \\
\hline Tubería 1" & $88 \mathrm{~m}$ & 4,200 & 369,600 \\
\hline Codos & 8 & 9,850 & 78,800 \\
\hline T's & 24 & 12,500 & 300,000 \\
\hline Tanques & 2 & - & $5,000,000$ \\
\hline Canaletas & 55 & 88,500 & $4,867,500$ \\
\hline Conexiones & 51 & 17,250 & 879,750 \\
\hline Bomba 1 & 1 & 523,000 & 523,000 \\
\hline Bomba 2 & 1 & 411,000 & 411,000 \\
\hline Personal & 3 & $1,200,000$ & $3,600,000$ \\
\hline Inversión total SCAPT (\$) & \multicolumn{3}{|c|}{$18,518,178$} \\
\hline $\begin{array}{l}\text { Agua lluvia } \\
\text { aprovechada anual }\left(\mathrm{m}^{3}\right)\end{array}$ & \multicolumn{3}{|c|}{1,537} \\
\hline Valor consumo por $\mathrm{m}^{3}(\$)$ & \multicolumn{3}{|c|}{8,010} \\
\hline Costo ahorrado anual (\$) & \multicolumn{3}{|c|}{$12,311,370$} \\
\hline
\end{tabular}

Tabla 7. Estudio de posibles costos y beneficios monetarios Fuente: Lista de precios de Pavco y tarifas EAAB.

De la tabla anterior es posible evidenciar que la inversión es recuperable en el primer año y medio de uso del sistema. Por otro lado, muestra un costo ahorrado de cerca de 12'311.370 de pesos colombianos anuales con tan solo usar dos de las bodegas más pequeñas de Corabastos S.A., teniendo en cuenta el costo del $\mathrm{m}^{3}$ de agua asociado al servicio de acueducto y alcantarillado de la EAAB. Si se proyectan los Scapt a las otras bodegas cuya área disponible para captación directa de agua lluvia sería mucho mayor a la contemplada anteriormente, se tendrían volúmenes de agua más grandes, por ende, beneficios económicos más representativos.
Es así como para futuras investigaciones se contemplaron secciones de techo verde sobre cada una de estas bodegas donde se propone un plan piloto en la bodega 26 que ha sido renovada con teja nueva termoacústica tipo sandwich, con techo verde de sistema tipo modular galocha autorregulado que tiene una capacidad de retener 20 litros por cada $\mathrm{m}^{2}$ [23]. Las plantas sugeridas son coralito, clavel chino, sedum y tulbagia. Al realizar la proyección a largo plazo, se obtienen 31 secciones con un área de $72.180 \mathrm{~m}^{2}$ que tendrían la capacidad de retener cerca de 1'443.600 litros de agua lluvia por cada evento masivo de precipitación (aguacero) en la zona de Corabastos.

Concibiendo los jardines verticales como coberturas verdes que deben ser promovidas se deduce que es viable compactar la mancha ecológica en el sector sur del HLV de la mano con actividades de reforestación en el parque Cayetano Cañizares, lo que permite incentivar el regreso de la biodiversidad característica en la zona y mitigar los impactos de la fragmentación en los humedales.

Como lo menciona [18], experiencias exitosas en ciudades como Tianjin, París y Ciudad de México son un referente importante para los escenarios actuales y la dinámica urbana de desarrollo que presenta una ciudad como Bogotá. Es decir, estas iniciativas son indispensables para aminorar los impactos ambientales negativos sobre los servicios ecosistémicos de todos los humedales del Distrito en escenarios futuros como la construcción de la Avenida Longitudinal (ALO) donde se hace necesario integrar su construcción con estrategias precisamente enmarcadas en el crecimiento verde.

\section{CONCLUSIONES}

La evaluación de las potenciales áreas de captación para aplicar SUDS muestra un total de $5.016 \mathrm{~m}^{2} \mathrm{di}$ rigidos al almacenamiento pluvial y posterior uso en labores de lavado y riego de jardines verticales y por otro lado, un total de $72.180 \mathrm{~m}^{2}$ dirigidos a la consolidación de techos verdes, lo que representa aproximadamente el 18,3\% de la totalidad del área de Corabastos.

Dada la continuidad de eventos de inundación desde 1921, se concluye que paralelo a las obras asociadas al acueducto y alcantarillado se deben considerar factores como la inadecuada gestión de los residuos sólidos, el cambio climático y la baja capacidad de retención de los humedales Techo, El Burro y La Vaca, que en últimas perpetúan el riesgo 
de inundación en la zona y abre las puertas a la aplicación de alternativas como los SUDS.

Después de realizar el análisis de oferta y demanda se muestra una cantidad total aproximada anual de $1.537 \mathrm{~m}^{3}$ de agua lluvia que se podrían aprovechar sin déficit de marzo a noviembre para cubrir los requerimientos de las enredaderas en los jardines verticales y el agua sobrante en usos asociados a limpieza de sanitarios y lavado de fachadas y pisos; lo que resalta la posibilidad de uso en el colegio al interior de Corabastos e incluso alimentar el caudal de la laguna de sedimentación para su posterior circulación por el biofiltro, y de esta manera cubrir el déficit hídrico que presenta el espejo de agua del HLV en temporadas secas.

Los sistemas urbanos de drenaje sostenible son excelentes herramientas para minimizar el impacto urbanístico, promueven buenas prácticas dirigidas al uso eficiente del recurso hídrico para lugares como la zona de influencia del HLV y del resto de humedales del Distrito. Con el caso particular de la localidad de Kennedy, que además de tener riesgo de inundación presenta índices negativos de calidad del aire, más en el HLV con la afluencia de vehículos de carga pesada, es allí, donde al robustecer la matriz vegetal de las zonas aledañas a los humedales con corredores ecológicos basados en jardines verticales o techos verdes, da como resultado enormes beneficios paisajísticos, ecológicos, sociales, entre otros.

\section{RECOMENDACIONES}

Incluir dentro del plan de mantenimiento de Corabastos, el monitoreo del sistema, donde se defina un rango de tiempo para vaciar los tanques y de esta forma alimentar el caudal de la laguna de sedimentación.

Considerar la adecuación de trampa anteriores y mallas de acero inoxidable al sistema de captación y almacenamiento de agua lluvia con el fin de proteger la calidad del agua lluvia colectada y evitar la proliferación de microorganismos y vectores, aunado a esto también es importante considerar posibles pérdidas por evaporación para más exactitud del balance hídrico.

Para próximos estudios, alineados con la presente propuesta es necesario continuar con la estimación de los costos asociados a la instalación y operación de los SUDS abordados. Así mismo, proyectar e incentivar los SUDS enmarcados en el crecimiento verde al resto de humedales del Distrito [24], lo que resalta aquellas unidades ecológicas que se vean intervenidas con la futura construcción de la Avenida Longitudinal (ALO).

\section{AGRADECIMIENTOS}

A la administración del Parque Ecológico Distrital Humedal La Vaca y a la oficina de Infraestructura y Medio Ambiente de Corabastos por su apoyo en las actividades de investigación.

\section{REFERENCIAS}

[1] R. Dawson, T. Ball, J. Werritty, A. Werritty, J. Hall y N. Roche, "Assessing the effectiveness of non-structural flood management measures in the Thames Estuary under conditions of socio-economic and environmental change," Global Environmental Change, vol. 21, $\mathrm{n}^{\circ} 2$, pp. 628-646, 2011.

[2] P. Gourbesville, "Urban flooding and Resilience: concepts and needs," en Asamblea General de EGU, Viena, Austria, 2012, p. 14282.

[3] V. Pappalardo y D. La Rosa, "Policies for Sustainable Drainage Systems in urban contexts within performance-based planning approaches," Sustainable Cities and Society, 2019.

[4] A. Massoudieh, M. Maghrebi, B. Kamrani, C. Nietch , M. Tryby, S. Aflaki y S. Panguluri, “A flexible modeling framework for hydraulic and water quality performance assessment of stormwater green infrastructure," Environmental Modelling \& Software, vol. 92, pp. 57-53, 2017.

[5] L. Ahiablame, B. Engel y I. Chaubey, "Representation and evaluation of low impact development practices with L-THIA-LID: An example for site planning," Reinar. Pollut, vol. 1, $\mathrm{n}^{\circ} 2$, pp. 1-13, 2012.

[6] C. Zamudio, "Gobernabilidad sobre el recurso hídrico en Colombia: entre avances y retos," Revista Gestión y Ambiente, vol. 15, n³, pp. 99-112, diciembre 2012. 
[7] Y. Carvajal, “Inundaciones en Colombia. ¿Estamos preparados para enfrentar la variabilidad y el cambio climático?," Revista Nacional de Investigación Memorias, vol. 9, $\mathrm{n}^{\circ}$ 16, $\mathrm{pp}$. 105-119, 2011.

[8] J. Ballén, M. Galarza y R. Ortiz, "Sistemas de Aprovechamiento de Agua Lluvia," en Seminário Iberoamericano sobre Sistemas de Abastecimento Urbano de Água João Pessoa (Brasil), 2006.

[9] C. Rojas, B. De Meulder y K. Shannon, "Water urbanism in Bogota. Exploring the potentials of an interplay between settlement patterns and water management," Habitat International, n 48, pp. 177-187, 2015.

[10] S. Perales y I. Doménech, "Los sistemas urbanos de drenaje sostenible: una alternativa a la gestión del agua de lluvia," 2008.

[11] S. Perales y I. Doménech, “Los sistemas urbanos de drenaje sostenible una alternativa a la gestión del agua lluvia," Valenica, 2008.

[12] A. Vásquez, "Infraestructura verde, servicios ecosistémicos y sus aportes para enfrentar el cambio climático en ciudades: el caso del corredor ribereño del río Mapocho en Santiago de Chile," Revista de Geografía Norte Grande, $\mathrm{n}^{\circ}$ 63, pp. 63-86, 2016.

[13] Secretaría Distrital de Ambiente, Sistemas urbanos de drenaje sostenible, Bogotá, 2011.

[14] D. Barragán y A. Sierra, Las inundaciones en Kennedy (Barrio Tierra Buena) afrontadas desde el diseño de información, Proyecto, Facultad de Arquitectura y Artes, Diseño Gráfico, Universidad Piloto de Colombia, Bogotá, 2013.

[15] Alcaldia Mayor de Bogotá. (2012), Plan Ambiental Local de Kennedy. [En línea]. Disponible en: http://www.ambientebogota. gov.co/documents / 10157/2883162/PAL+ Kennedy+2013-2016.pdf.

[16] DANE, “Población 2007,” Bogotá, 2007.

[17] EEAB, "Plan de manejo ambiental del humedal la Vaca," Bogotá, 2009.

[18] G. González, El impacto de la dinámica urbana sobre los humedales de Bogotá (Colombia). Análisis, modelización y propuestas en el marco del crecimiento verde, Tesus, Universidad de Zaragoza, España, 2018.

[19] T. Van der Hammen, Plan Ambiental de la cuenca alta del río Bogotá: análisis y orientación para el ordenamiento territorial. CAR, Bogotá, Bogotá: CAR, 1998.

[20] G.A. Forero Buitrago, "Dimensionamiento de canales y embalses para conducir y almacenar agua lluvia para abastecer la población de Altos de Cazucá (Soacha-Colombia) utilizando DEM," Revista de Tecnología, Vol. 16, $\mathrm{n}^{\circ}$ 1, pp. 113-128, 2018.

[21] Idiger, Hemeroteca de emergencias de Bogotá, Bogotá, 2019.

[22] Idiger, (2018), “Caracterización general de escenario de riesgo de inundación por desbordamiento," 27 noviembre. [En línea]. Dispobible en: http://www.idiger.gov.co/ rinundacion.

[23] CYPE Ingenieros S, A., "Generador de precios Colombia," 2019. [En línea]. Disponible en: http://www.colombia.generadordeprecios. info/obra_nueva/Cubiertas/Planas/No_transitables_no_ventiladas/QAD050_Cubierta_ verde_sistema_Urbanscape_.html

[24] Secretaría Distrital de Ambiente, (2018) "Red de Monitoreo de Calidad del Aire de Bogotá," [En línea]. Disponible en: http://201.245.192.252:81/. 